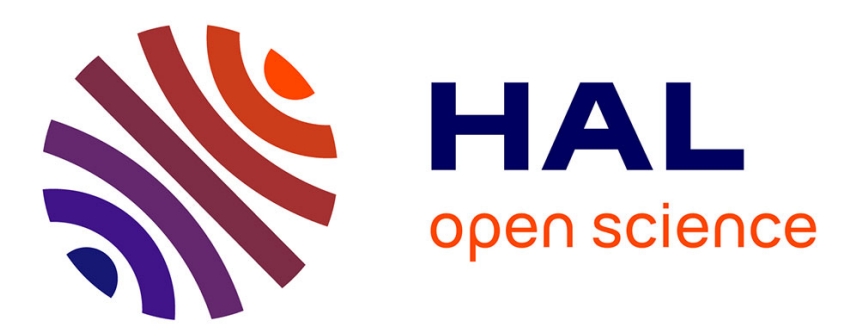

\title{
Électroréflexion : mesures et systèmes d'asservissement
}

\author{
A. Jolivet, R. Garrigos, R. Kofman
}

\section{To cite this version:}

A. Jolivet, R. Garrigos, R. Kofman. Électroréflexion: mesures et systèmes d'asservissement. Revue de Physique Appliquée, 1972, 7 (4), pp.403-407. 10.1051/rphysap:0197200704040300 . jpa-00243645

\section{HAL Id: jpa-00243645 https://hal.science/jpa-00243645}

Submitted on 1 Jan 1972

HAL is a multi-disciplinary open access archive for the deposit and dissemination of scientific research documents, whether they are published or not. The documents may come from teaching and research institutions in France or abroad, or from public or private research centers.
L'archive ouverte pluridisciplinaire HAL, est destinée au dépôt et à la diffusion de documents scientifiques de niveau recherche, publiés ou non, émanant des établissements d'enseignement et de recherche français ou étrangers, des laboratoires publics ou privés. 
Classification

Physics Abstracts

$18.10,05.30$

\title{
ÉLECTRORÉFLEXION : MESURES ET SYSTÈMES D'ASSERVISSEMENT
}

\author{
A. JOLIVET, R. GARRIGOS et R. KOFMAN \\ Laboratoire d'Electro-optique, Parc Valrose, Nice
}

(Reçu le 20 juin 1972, révisé le 27 juillet 1972)

\begin{abstract}
Résumé. - On décrit les deux systèmes d'asservissement intégrés dans un appareillage de mesures d'électroréflexion : l'un pour maîtriser en amplitude et en forme l'excitation à l'interface métal-électrolyte, l'autre pour stabiliser le courant anodique moyen du photomultiplicateur. Les grandeurs mesurées ainsi que leur signe sont déterminés sans ambiguïté. De récents résultats obtenus avec l'or et l'argent sont donnés en illustration.
\end{abstract}

Abstract. - Two servo-mechanisms used for electroreflectance measurements are described. The first one is used to act upon the amplitude and the shape of the excitation at the metal-electrolyte interface, the second one to keep constant the mean anodic current of the photomultiplier. The measured quantities and their signs are unambiguously determinated. Recent results on gold and silver are given as examples.

I. Introduction. - La connaissance du coefficient de réflexion $R$ d'un solide suffit en principe pour déterminer son facteur de permittivité complexe $\varepsilon=\varepsilon_{1}+i \varepsilon_{2}$ à partir duquel s'interprète sa structure en bandes d'énergie. Cependant la faible modulation des spectres obtenus expérimentalement rend les conclusions assez douteuses [1].

Par contre, un champ électrique intense, de l'ordre de $10^{5} \mathrm{~V} . \mathrm{cm}^{-1}$, créé au voisinage de la surface de ce solide à l'aide d'une solution électrolytique et d'une différence de potentiel $u$ de l'ordre du volt, module le coefficient de réflexion $R$ : la grandeur mesurée expérimentalement $(1 / R)(\partial R / \partial u)$ permet d'atteindre les dérivées

$$
\frac{\partial \varepsilon_{1}}{\partial u} \quad \text { et } \quad \frac{\partial \varepsilon_{2}}{\partial u}
$$

Le spectre de cette dernière dérivée, en fonction de l'énergie du photon, possède souvent une richesse en extrémums, changements de signe et points d'inflexion, qui ne se retrouve pas dans le spectre de $\varepsilon_{2}$.

Dans le cas des semi-conducteurs, on sait [2] que les énergies signalées par ces spectres sont en relation avec les transitions optiques interbandes en des points particuliers de la zone de. Brillouin.

Dans le cas des métaux, en particulier l'or et l'argent, l'explication théorique est rendue plus complexe par la faible pénétration du champ électrique appliqué par rapport à celle du champ électrique de l'onde lumineuse.

Cet exposé n'a pas pour but de faire connaître une nouvelle théorie, mais d'en préparer et d'en affermir les bases : il indique d'abord comment on peut résoudre assez simplement deux difficultés techniques, puis il précise la nature et le signe des grandeurs mesurées, enfin il donne quelques résultats expérimentaux.
II. Des appareillages. - Lorsque les longueurs d'onde $(0,17$ à $0,95 \mu \mathrm{m})$ de la lumière filtrée par le monochromateur (Jobin-Yvon HRS 2) défilent régulièrement avec le temps, le flux lumineux reçu par le photomultiplicateur varie dans d'énormes proportions pour diverses raisons dont la présence de raies intenses dans le spectre d'émission de la source (lampe au xénon OSRAM XBO $150 \mathrm{~W} / 4$ ). A cela s'ajoute la réponse non linéaire en longueur d'onde du photomultiplicateur.

Le meilleur moyen de s'affranchir de telles variations consiste à stabiliser le courant anodique moyen du photomultiplicateur en utilisant l'écart de fréquence entre les phénomènes gênants et lents d'une part, et les phénomènes étudiés plus rapides d'autre part.

Un premier système d'asservissement réalise cette stabilisation en agissant sur la tension d'alimentation et par conséquent sur le gain du photomultiplicateur.

Pour essayer de maîtriser la différence de potentiel $u$ à la jonction échantillon métallique-électrolyte, il faut plonger dans ce dernier deux électrodes : une électrode au calomel parcourue par des courants réduits au minimum (résistance du circuit de l'ordre de $10^{12} \Omega$ ) et destinée à relever le potentiel de l'électrolyte (avec une certaine correction inconnue mais constante lors de nos mesures), et une contre-électrode de grande surface en or destinée à amener le courant indépendamment de sa propre polarisation.

Un second système d'asservissement commande le courant injecté à travers la contre-électrode de telle sorte que l'électrode au calomel présente par rapport à l'échantillon la différence de potentiel $u$ fournie par un générateur de signaux.

Ces deux systèmes d'asservissement sont inclus, suivant le schéma de la figure 1, dans l'appareillage comprenant un générateur de signaux avec un détec- 
teur synchrone, un monochromateur avec sa source, un photomultiplicateur avec son alimentation, et deux enregistreurs (non figurés).

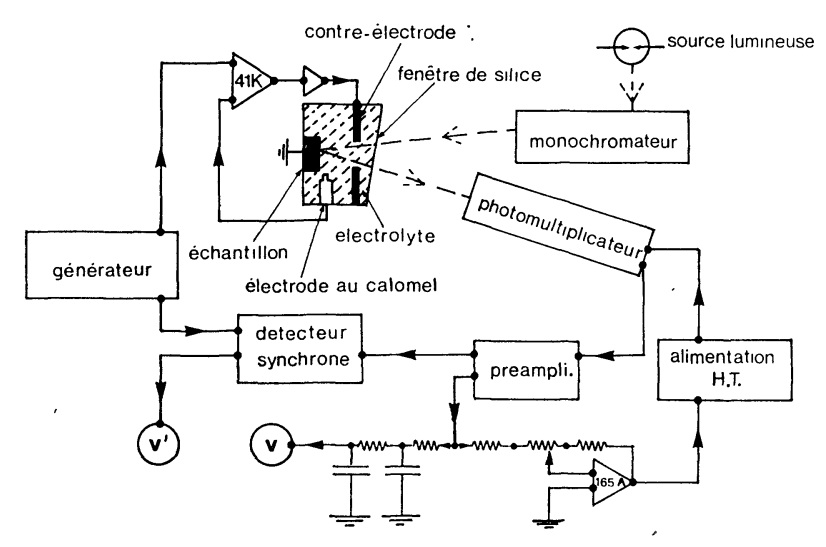

Fig. 1. - Dispositif expérimental : la mesure de la dérivée relative du coefficient de réflexion se déduit simplement de la valeur du rapport des deux tensions $v^{\prime}$ et $v$.

II. 1 STABILISATION DU COURANT ANODIQUE MOYEN DU PHOTOMULTIPLICATEUR. - L'alimentation (Mesco HTT 2503) est normalement stabilisée en tension : c'est-à-dire qu'une fraction -de la tension de sortie, comparée à une référence, fournit un signal d'erreur qui commande l'ajustement de la tension de sortie. Grâce à une légère modification interne, il est possible de comparer à la référence, la tension, convenablement amplifiée, délivrée par le préamplificateur (PAR 184) laquelle est proportionnelle au courant anodique du photomultiplicateur (EMI 9659 QB). L'amplificateur opérationnel $165 \mathrm{~A}$ (Analog Devices) procure un gain stable et ajustable; il travaille avec une tension de sortie de $13 \mathrm{~V}$ et une tension de commande $v$ qui varie entre 10 et $400 \mathrm{mV}$ suivant l'intensité moyenne choisie : 0,1 à $4 \mu \mathrm{A}$. Un correcteur de phase évite les oscillations.

II. 2 ASSERVISSEMENT DE LA DIFFÉRENCE DE POTENTIEL. - L'échantillon métallique (sous forme de monocristal, polycristal, film) dont une face est en contact avec l'électrolyte, est mis au potentiel zéro. Une électrode conventionnelle au calomel relève le potentiel de l'électrolyte qui est asservi au signal fourni par le générateur (Wavetek 142) au moyen d'un amplificateur opérationnel $41 \mathrm{~K}$ (Analog Devices) suivi d'un amplificateur de courant (deux transistors complémentaires de SGS : BF X 38 et BF Y 56 A) lequel alimente la contre-électrode. L'excursion en tension peut atteindre $\pm 12 \mathrm{~V}$.

III. Des grandeurs mesurées et de leur signe. La question du signe repose sur une convention qui doit être énoncée d'autant plus clairement qu'elle est arbitraire. Avec notre montage, cette convention s'impose naturellement : le potentiel $u(t)$ est celui de l'électrode au calomel par rapport à l'échantillon. Il est de la forme :

$$
u(t)=u_{0}+u_{1} \cos \omega t
$$

avec

$u_{1}>0, \quad \omega=2 \pi v, \quad v \in[2,1000 \mathrm{~Hz}], \quad T=1 / v$.

Notre dispositif expérimental accompagnant le détecteur synchrone (PAR 124) permet l'étude des dérivées du coefficient de réflexion $R(u, \lambda)$ par rapport à $u$, au voisinage de $u_{0}$ et en fonction de $\lambda$ (Fig. 2).

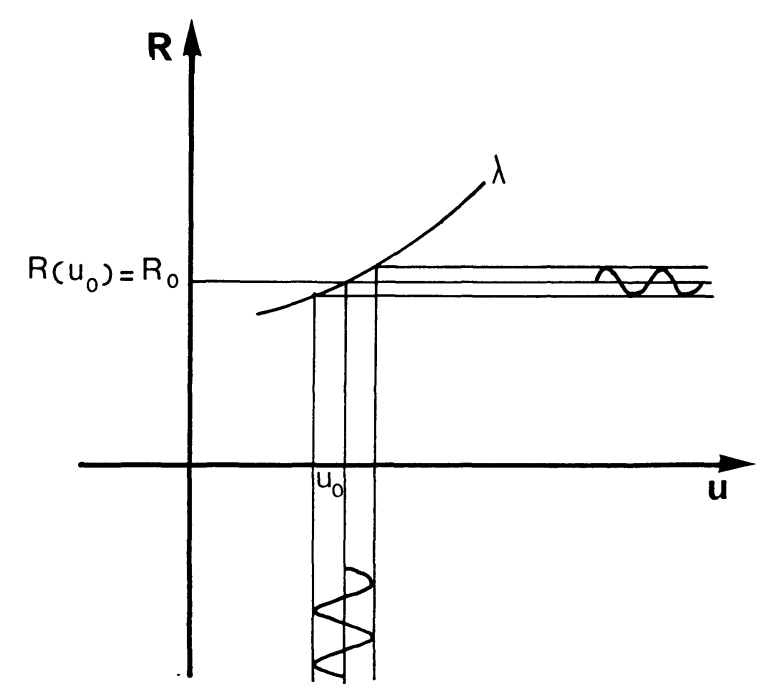

FIG. 2. - Pour une longueur d'onde donnée $\lambda$, le coefficient de réflexion $R$ est une fonction dérivable de la différence de potentiel $u$ à la jonction métal-électrolyte.

Pour une longueur d'onde $\lambda$ donnée, $R$ est fonction du temps par l'intermédiaire de $u(t)$ et se développe en série de Taylor:

$$
\begin{aligned}
R(t)=R_{0}+\frac{\partial R}{\partial u} u_{1} \cos \omega t+ & \frac{\partial^{2} R}{\partial u^{2}} \frac{u_{1}^{2}}{2} \cos ^{2} \omega t+ \\
& +\frac{\partial^{3} R}{\partial u^{3}} \frac{u_{1}^{3}}{6} \cos ^{3} \omega t+\cdots
\end{aligned}
$$

La tension moyenne $v$ recueillie à la sortie du préamplificateur (dont l'inversion de phase neutralise celle du photomultiplicateur) est proportionnelle à la moyenne de $R$ qui se confond avec $R_{0}$.

$$
v=K \frac{1}{T} \int_{0}^{T} R(t) \mathrm{d} t=K R_{0} .
$$

En effet, toutes les puissances impaires de $\cos \omega t$ donnent une intégrale nulle tandis que la contribution des puissances paires reste négligeable devant celle de $R_{0}$, comme on s'y attend a priori et comme on le vérifie expérimentalement, par exemple avec le résultat numérique donné en IV.3, la contribution du terme contenant $\cos ^{2} \omega t$ est inférieure à $10^{-4}$ fois celle de $R_{0}$.

III.1 DÉTECTION SYNCHRONE A LA FRÉQUENCE FONDAMENTALE. - On appelle $\psi(t)$ le gain variable 
(en fonction du temps) du détecteur synchronisé sur le générateur avec un retard de phase nul (Fig. 3).
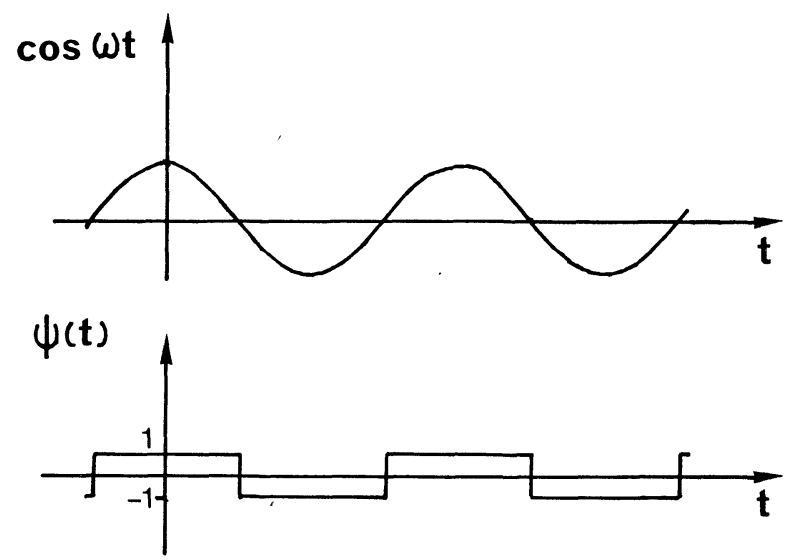

FIG. 3. - La sinusoïde cos $\omega t$ est parfaitement détectée par le détecteur synchrone lorsqu'elle est en phase avec son gain $\psi(t)$.

La tension $v^{\prime}$ recueillie à la sortie du détecteur synchrone est donnée par la moyenne temporelle du produit $R(t) \cdot \psi(t)$ où seule la dérivée première donne une intégrale non nulle.

$$
\begin{aligned}
v^{\prime}=K \frac{1}{T} & \int_{-1 T / 4}^{+3 T / 4} \psi(t) \cdot R(t) \cdot \mathrm{d} t= \\
& =K \frac{2}{T} \int_{-T / 4}^{+T / 4} \frac{\partial R}{\partial u} u_{1} \cos \omega t \mathrm{~d} t=K \frac{2}{\pi} u_{1} \frac{\partial R}{\partial u} .
\end{aligned}
$$

Il s'agit bien du même coefficient $K$, parce que l'on prend la précaution d'ajuster les gains de telle sorte que $v^{\prime}=v$ pour un signal en créneaux $y(t)$ injecté à l'entrée du préamplificateur, le détecteur fonctionnant en « large bande " $\left(y(t)=-y_{0}\right.$ sur une demi-période et $y(t)=0$ sur l'autre demi-période). Ce coefficient $K$, inconnu ici, s'élimine en formant le rapport $v^{\prime} / v$.

$$
\frac{1}{R_{0}} \frac{\partial R}{\partial u}=\frac{\pi}{2} \frac{1}{u_{1}} \frac{v^{\prime}}{v} .
$$

Pratiquement on enregistre $v^{\prime}$ en maintenant constants $u_{1}$ et $v$ à l'aide des systèmes d'asservissement déjà décrits et en faisant défiler lentement des longueurs d'onde $\lambda$ (durée d'enregistrement : 15 à $30 \mathrm{mn}$ ). Cet enregistrement traduit donc, à un coefficient (constant, positif et connu) près, la dérivée première relative du coefficient de réflexion :

$$
\frac{1}{R_{0}} \frac{\partial R}{\partial u}
$$

III. 2 DÉTECTION SYNCHRONE ȦUX FRÉQUENCES HARMONIQUES. - Les phénomènes observés à la fréquence fondamentale sont déjà assez petits; ceux correspondant aux fréquences harmoniques le sont bien davantage ou même complètement inobservables, cela montre que le graphe $R(U)$ présente une très faible courbure et justifie a posteriori l'hypothèse de la dérivabilité.
III.2.a Fréquence double. - Le gain $\psi(t)$ varie à la fréquence $2 v$ avec un retard de phase de $90^{\circ}$. A la sortie du détecteur synchrone on recueille une tension $v^{\prime \prime}$ qui est proportionnelle (toujours même coefficient $K$ ) à la dérivée seconde de $R$.

$$
\begin{aligned}
v^{\prime \prime}= & K \frac{1}{T} \int_{-7 T / 8}^{+7 T / 8} \psi(t) \cdot R(t) \cdot \mathrm{d} t= \\
& =K \frac{4}{T} \int_{-T / 8}^{+T / 8} \frac{\partial^{2} R}{\partial u^{2}} \frac{u_{1}^{2}}{2} \cos ^{2} \omega t \mathrm{~d} t=K \frac{u_{1}^{2}}{2 \pi} \frac{\partial^{2} R}{\partial u^{2}} !
\end{aligned}
$$

En formant le rapport $v^{\prime \prime} / v$ il vient :

$$
\frac{1}{R_{0}} \frac{\partial^{2} R}{\partial u^{2}}=2 \pi \frac{1}{u_{1}^{2}} \frac{v^{\prime \prime}}{v} .
$$

III.2.b Fréquence triple. - De façon analogue quand $\psi(t)$ varie à la fréquence $3 v$ avec un retard de phase de $180^{\circ}$ on recueille une tension $v^{\prime \prime \prime}$ proportionnelle à la dérivée troisième :

$$
\begin{aligned}
v^{\prime \prime \prime} & =K \frac{1}{T} \int_{-1 T / 12}^{+11 T / 12} \psi(t) \cdot R(t) \cdot \mathrm{d} t \\
& =K \frac{6}{T} \int_{-T / 12}^{+T / 12} \frac{\partial^{3} R}{\partial u^{3}} \frac{u_{1}^{3}}{6} \cos ^{3} \omega t=K \frac{1}{12 \pi} u_{1}^{3} \frac{\partial^{3} R}{\partial u^{3}}
\end{aligned}
$$

etc.

IV. Exemples de l'or et de l'argent. - Les échantillons sont taillés dans le métal massif puis polis.

IV.1 Electrolyte et excitation. - Avec l'or le choix de l'électrolyte importe peu : nous avons utilisé l'acide perchlorique $0,1 \mathrm{M}$. Par contre l'argent est très sensible à l'oxydation : nous avons préféré l'acide oxalique $0,1 \mathrm{M}$. L'enregistrement à très basse fréquence $(0,02 \mathrm{~Hz}$ par exemple) de la densité de courant $i$ traversant la jonction échantillon-électrolyte en fonction de la différence de potentiel $u$, permet de choisir un intervalle d'excursion en tension où ne se manifestent ni oxydation, ni réduction : seul le courant de fuite de la jonction se superpose au courant de charge de sa capacité.

Les figures 4 et 5 montrent, pour chacun des échan-

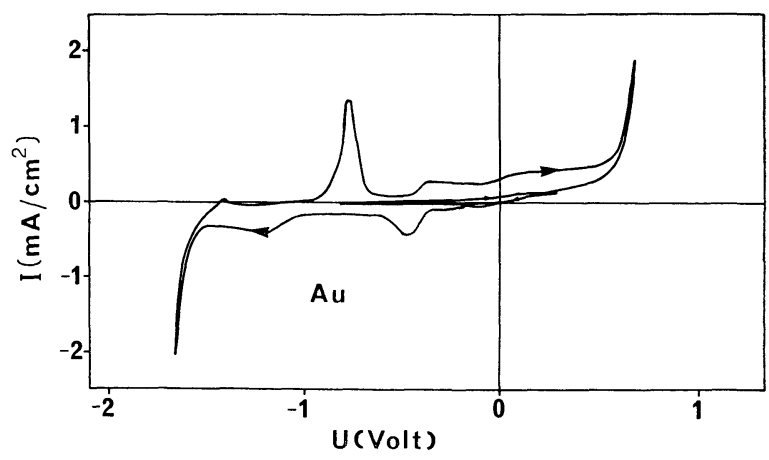

FIg. 4. - Deux cycles de la densité de courant $i$ en fonction de la tension $u$, décrits en $50 \mathrm{~s}$ avec une tension parfaitement sinusoïdale : le plus petit est parcouru avec la même excursion en tension que celle utilisée pour toutes les mesures, le plus grand fait apparaître les phénomènes d'oxydation et de réduction, avec dégagement d'hydrogène sur l'échantillon en haut à droite. 


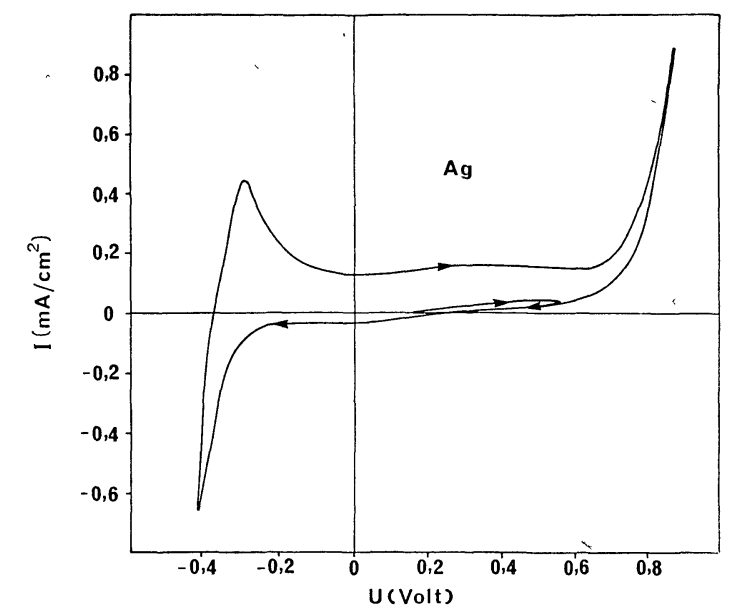

FIG. 5. - Deux cycles analogues aux précédents ; on remarque combien l'argent réagit plus facilement que l'or et n'autorise pas une aussi grande excursion en tension :' $400 \mathrm{mV}$ au lieu de $1100 \mathrm{mV}$.

tillons, l'intervalle choisi pour les mesures, inscrit à l'intérieur d'un intervalle plus grand.

IV. 2 VARIATION DE $\left(1 / R_{0}\right)(\partial R / \partial u)$ AVEC LA LONGUEUR D'ONDE $\lambda$. - Il faut choisir une fréquence d'excitation $v$ assez basse, pour une raison qui apparaîtra plus loin, mais aussi assez éloignée de la fréquence de résonance de la stabilisation en courant $(6 \mathrm{~Hz})$, et encore de certains sous-harmoniques du secteur : l'intervalle $32-34 \mathrm{~Hz}$ semble optimal.

La détection à la fréquence fondamentale donne la variation de $v^{\prime}$ en fonction de $\lambda$ : il suffit alors d'étalonner l'enregistrement sur un seul point (un pic) pour avoir $\left(1 / R_{0}\right)(\partial R / \partial u)$ en fonction de $\lambda$. Le retard de phase qui reste voisin de $165^{\circ}$ correspond à un signe négatif et à une avance résiduelle de $15^{\circ}$. Les figures 6 et 7 reproduisent deux de ces enregistrements.

IV. 3 VARIATION DE $\left(1 / R_{0}\right)(\partial R / \partial u)$ AVEC LA FRÉQUENCE D'EXCITATION $v$. Pour cette étude il est nécessaire de bloquer à la fois la longueur d'onde $\lambda$

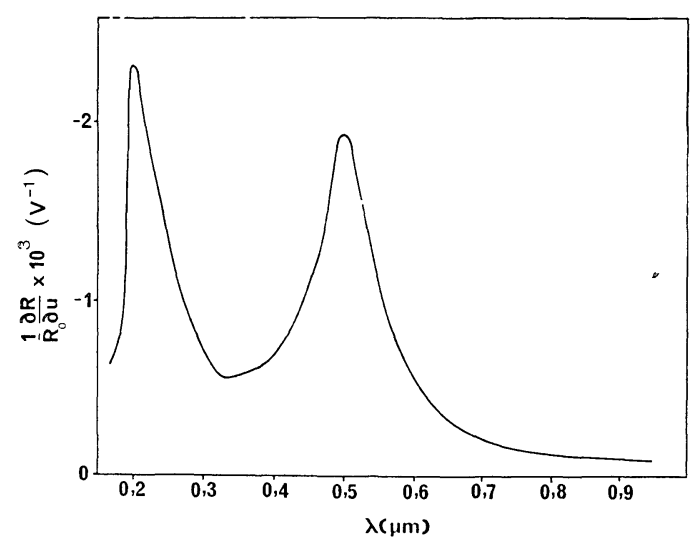

Fig. 6. - Dérivée première relative du coefficient de réflexion de l'or en présence d'une solution aqueuse d'acide perchlorique $0,1 \mathrm{M}$, en fonction de la longueur d'onde de la lumière. Remarquer les deux pics à 0,210 et $0,495 \mu \mathrm{m}$.

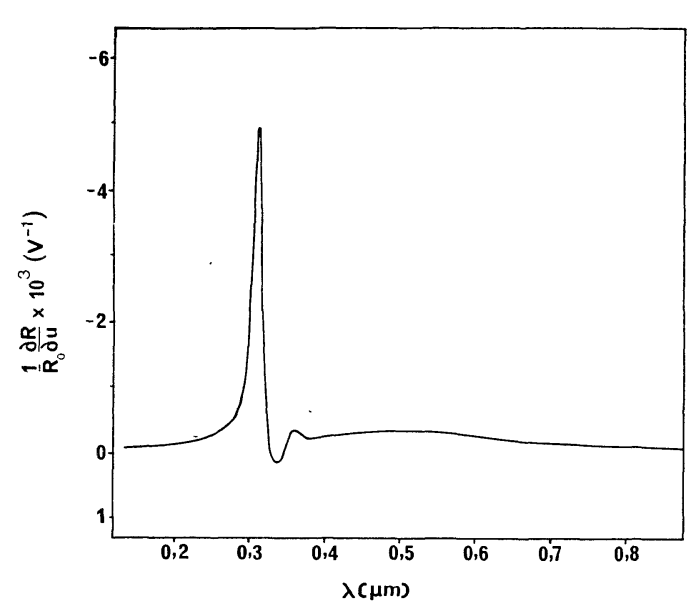

Fig. 7. - Dérivée première relative du coefficient de réflexion de l'argent en présence d'une solution aqueuse d'acide oxalique $0,1 \mathrm{M}$, en fonction de la longueur d'onde de la lumière. Noter le pic à $0,315 \mu \mathrm{m}$.

(sur une réponse maximale) et la tension d'alimentation du photomultiplicateur (sur celle correspondant au courant moyen anodique choisi). La détection synchrone à la fréquence fondamentale qui varie alors entre $2 \mathrm{~Hz}$ et $1 \mathrm{kHz}$ fait ressortir, comme le montre la figure 8 , des variations du signal assez semblables pour l'or et l'argent.

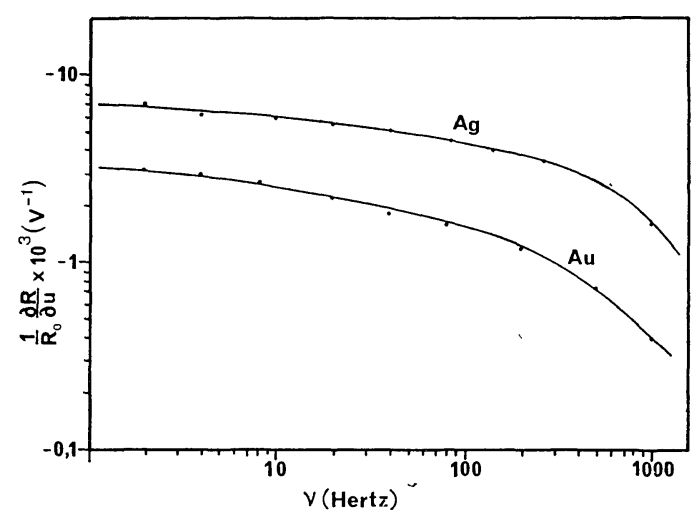

FIG. 8. - Dérivées relatives des coefficients de réflexion de l'or et de l'argent en fonction de la fréquence d'excitation $v$; noter que celle de l'argent est environ deux fois plus grande que celle de l'or.

De plus, pour l'argent, nous avons relevé $v^{\prime}$ et aussi $v^{\prime \prime}$ à la fréquence de $34 \mathrm{~Hz}(\lambda=0,315 \mu \mathrm{m})$. Cela donne :

$\frac{1}{R_{0}} \frac{\partial R}{\partial u}=-7,2 \times 10^{-3} \mathrm{~V}^{-1}$ (précision : $\pm 5 \%$ )

$\frac{1}{R_{0}} \frac{\partial^{2} R}{\partial u^{2}}=-6,3 \times 10^{-3} \mathrm{~V}^{-2}$ (précision : $\pm 20 \%$ ).

V. Conclusion. - Concernant la référence des potentiels, la nature des circuits électriques nous a induit à choisir une convention contraire aux habitudes des électrochimistes ; en conséquence chacun tiendra 
compte d'un simple changement de signe pour le passage d'une convention à l'autre.

Concernant l'objet principal de ces mesures nous sommes amenés à préciser que la grandeur très souvent désignée par $\Delta R / R$ [1], [2], [3], [4], [5] doit, plus exactement, s'entendre comme celle de :

$$
\frac{1}{R_{0}} \frac{\partial R}{\partial u} \Delta u \quad \text { pour } \quad \Delta u=1 \mathrm{~V}(\mathrm{ou}-1 \mathrm{~V})
$$

où la dérivée relative est calculée conformément à la formule (1).

Quant au phénomène il reste ce qu'il est : pour la plus grande partie des spectres étudiés et notamment pour les réponses maximales on remarque que c'est un champ électrique correspondant à un excès d'électrons dans le métal (or ou argent) qui coïncide avec une diminution de son coefficient de réflexion.

Abstraction faite du signe, la modulation relative du coefficient de réflexion reste faible puisqu'elle est inférieure à $10^{-2} \mathrm{~V}^{-1}$.

Par ailleurs les spectres obtenus montrent des accidents bien mieux localisables, en longueur d'onde, que ceux du coefficient de réflexion lui-même. Pour l'or par exemple l'extrémum à $0,495 \mu \mathrm{m}$ correspondant au seuil des transitions interbandes

$$
\left(Q_{3}+Q_{4}\right)^{5} \rightarrow E_{\mathrm{F}}\left(Q_{3}+Q_{4}\right)^{6}
$$

(Notation Rapw [8]) est plus net sur le spectre de $(1 / R)(\partial R / \partial u)$ que sur celui de $R$; quant à l'autre extrémum à $0,210 \mu \mathrm{m}$ il n'est pas visible sur le spectre de $R$.

\section{Bibliographie}

[1] Cooper (B. R.), Ehrenreich (H.) and Phillip (H. R.), Phys. Rev., 1965, 138, A 494.

[2] Cardona (M.), Shaklee (K. L.) and Pollak (F. M.), Phys. Rev., 1966, 154, 696.

[3] Feinleib (J.), Phys. Rev. Letters, 1966, 16, 1200.

[4] Hansen (W. N.) et Prostak (A.), Phys. Rev, 1968, 174,500 .
[5] Buckman (A. B.) èt Bashara (N. M.), Phys. Rev., $1968,174,719$.

[6] Garrigos (R.), Kofman (R.), Jolivet (A.) et DonnaDIEU (A.), C. R. Acad. Sci., Paris, 1971, 272B, 1078.

[7] Garrigos (R.), Kofman (R.), Jolivet (A.) et Richard (J.), Il Nuovo Cimento, 1972, 8B, 1, 242.

[8] Christensen (N. E.) et Seraphin (B. O.), Phys. Rev., 1971, B 4, 3321. 